\title{
Prognostic factors for recurrence and survival in uterine leiomyosarcoma: Korean single center experience with 50 cases
}

\author{
E Sun Paik ${ }^{*}$, Jae Hong Kang ${ }^{*}$, Jihye Kim, Yeon-Joo Lee, Chel Hun Choi, Tae-Joong Kim, Byoung-Gie Kim, \\ Duk-Soo Bae, Jeong-Won Lee \\ Department of Obstetrics and Gynecology, Samsung Medical Center, Sungkyunkwan University School of Medicine, Seoul, Korea
}

\section{Objective}

The aim of this study was to determine the possible prognostic factors in patients with uterine leiomyosarcoma (LMS).

\section{Methods}

This study retrospectively investigated 50 patients with uterine LMS treated at the Samsung Medical Center between 2001 and 2017. To analyze the prognostic significance of factors for recurrence-free survival (RFS), overall survival (OS), and survival after recurrence, the log-rank test and Cox proportional hazards model were used for univariate and multivariate analysis.

\section{Results}

Of the 50 patients, $30(60.0 \%)$ experienced recurrence and $16(32.0 \%)$ died within a median follow-up period of 21 (range, 3-99) months. Multivariate analysis revealed that older age, absence of residual tumor after surgery, lower mitotic count, and a history of adjuvant radiotherapy at first treatment were significantly associated with better RFS. Presence of residual tumor after surgery and severe nuclear atypia were associated with poor OS. In the analysis of survival after recurrence, hematogenous recurrence, severe nuclear atypia, and presence of residual tumor at primary surgery were significantly associated with worse prognosis. Notably, residual tumor status at primary surgery was associated with RFS, OS, and survival after recurrence.

\section{Conclusion}

We demonstrated the possible prognostic factors for RFS, OS, and survival after recurrence for patients with LMS. These results may provide useful information for patients with LMS.

Keywords: Uterine neoplasm; Leiomyosarcoma; Prognostic factor; Survival; Recurrence

\section{Introduction}

Uterine leiomyosarcoma (LMS) is a rare uterine cancer that accounts for approximately $1 \%$ of all uterine cancers $[1,2]$. However, because of its aggressive biology and resistance to chemotherapy, LMS causes approximately $70 \%$ of deaths caused by uterine malignant tumors $[3,4]$. The symptoms and presenting characteristics of uterine LMS are almost indistinguishable from those of uterine leiomyoma [1]. Palpable mass and abnormal vaginal bleeding followed by weight loss and general weakness are the most common symptoms of uterine LMS $[5,6]$. Intraoperatively, it is difficult to differentiate between LMS and leiomyoma based on visible evidence
Received: 2018.06.26. Revised: 2018.09.25. Accepted: 2018.10.23. Corresponding author: Jeong-Won Lee

Department of Obstetrics and Gynecology, Samsung Medical Center, Sungkyunkwan University School of Medicine, 81 Irwonro, Gangnam-gu, Seoul 06351, Korea E-mail: garden.lee@samsung.com https://orcid.org/0000-0002-6110-4909

${ }^{\star}$ These authors contributed equally to this work.

Articles published in Obstet Gynecol Sci are open-access, distributed under the terms of the Creative Commons Attribution Non-Commercial License (http://creativecommons. org/licenses/by-nc/3.0/) which permits unrestricted non-commercial use, distribution, and reproduction in any medium, provided the original work is properly cited.

Copyright $\odot 2019$ Korean Society of Obstetrics and Gynecology 


\section{Obstetrics \& Gynecology Science}

Vol. 62, No. 2, 2019

alone [7], and uterine LMS is often diagnosed by histologic evaluation of tumor specimens.

Surgical removal of tumors, including hysterectomy and/ or bilateral salpingo-oophorectomy, is the standard management for uterine LMS. However, surgical staging is regarded as less important because uterine LMS is known to have early hematogenous metastasis and rare lymphatic spread [8]. Recently, there has been an increase in the frequency of adjuvant chemotherapy and radiation therapy for the treatment of uterine LMS. However, adjuvant treatment has not shown a definite survival advantage [3].

Although in most cases, the tumor is limited to the uterus, it remains difficult to predict the disease course of uterine LMS. Stage is the strongest predictor of survival. Various other prognostic factors have previously been suggested for uterine LMS, but these remain controversial and the available data is limited $[9,10]$. We aimed to determine the possible prognostic factors for recurrence, overall survival (OS), and survival after recurrence in patients with uterine LMS.

\section{Materials and methods}

\section{Patients and treatment}

The medical records of 50 patients with uterine LMS, who were diagnosed and treated at Samsung Medical Center from 2001 to 2017, were retrospectively reviewed. Per the routine protocol in patients with uterine LMS, patients underwent total hysterectomy and salpingo-oophorectomy (either bilateral or unilateral). The decision to perform adjuvant therapy (chemotherapy/radiation therapy) was made on the basis of the physician's opinion and patient's situation. To treat recurrent disease, chemotherapy, radiotherapy, and/or surgical resection of tumor were considered, if feasible. Available histological slides were also reviewed by a gynecologic pathologist. LMS was histologically defined by the presence of 2 of the following 3 criteria: 1) significant nuclear atypia, 2) $>10$ mitotic counts per 10 high-power field (HPF), and 3) coagulative tumor cell necrosis [11]. The modified 2009 International Federation of Gynecology and Obstetrics (FIGO) staging for LMS was used.

Clinicopathologic, surgical, and survival data were retrospectively gathered from electronic medical records. Prognostic variables that were included in this study were age at primary diagnosis, tumor suspected as leiomyoma with clini- cal characteristics before surgery, FIGO stage, residual disease status after primary surgery, tumor size, symptoms at diagnosis, grade, nuclear atypia, mitotic count (per 10 HPF), history of adjuvant therapy after primary surgery (chemotherapy/radiotherapy), recurrence pattern (peritoneal/hematogenous), and treatment after recurrence. Characteristics were assigned to categories for descriptive purposes and statistical analysis.

Tumor size was recorded on the basis of the maximum dimension of the tumor at pathologic analysis. The tumor grade, nuclear atypia, and mitotic count were recorded on the basis of final pathologic reports. Symptoms at diagnosis were limited to those related to the uterine mass at the time of diagnosis. Recurrence patterns were categorized into peritoneal and hematogenous metastases. Peritoneal recurrences were defined as the recurrence of metastatic lesions in the peritoneum of the pelvis and abdominal areas. Hematogenous recurrences were defined as visceral metastases such as those in the liver and lung parenchyma.

Recurrence-free survival (RFS) was defined as the time of initial diagnosis to the date of recurrence, death, or loss to follow-up. OS was described as the time between diagnosis and the patient's death or loss to follow-up. Survival after recurrence was described as the time between the diagnosis of recurrence and the patient's death or loss to follow-up.

\section{Statistical analysis}

Summary statistics were used to describe the data. Median (range) or mean (standard deviation) was used to describe continuous variables. Categorical variables were presented as frequencies (percentages). After confirming normal distributions using the Shapiro-Wilk test, the Mann-Whitney test was used to compare median values, and the Student's t-test was used to compare mean values. Categorical variables were presented as frequencies (percentages). Fisher's exact test or $\chi^{2}$ test was used to analyze the distribution of characteristics. Survival curves were drawn by the KaplanMeier method and were compared using the log-rank test. Cox proportional hazards model was used for univariate and multivariate analyses to evaluate the prognostic significance of clinicopathologic features for RFS, OS, and survival after recurrence. For multivariate analysis, a stepwise backward elimination method was used. Variables that were significantly associated with RSF, OS, and survival after recurrence with a significance level of $P<0.10$ in univariate analysis were selected for possible inclusion in multivariate logistic regres- 


\section{Obstetrics \& Gynecology Science}

E Sun Paik, et al. Prognosis of uterine leiomyosarcoma

sion models, as it was previously suggested that this value could be used as an appropriate threshold [12]. Multivariate $P$-values were used to represent the significance of each feature. A 95\% confidence interval $(\mathrm{Cl})$ was used to quantify the correlation between survival time and each independent feature. All $P$-values were 2 -sided, and $P$-values $<0.05$ were regarded as statistically significant. All statistical analyses were accomplished using IBM SPSS ver. 21.0 (IBM Corp., Armonk, NY, USA).

\section{Results}

The clinicopathologic characteristics of 50 patients with uterine LMS are presented in Table 1. At the time of analysis, 30 patients (60.0\%) experienced recurrence, and 16 patients (32.0\%) died within a median follow-up period of 21 (range, 3-99) months. Median age was 48 (range, 31-72) years, and $60.0 \%$ of the patients had stage I disease. Median tumor size was 10 (range, 3.0-29.0) cm, and moderate atypia was the most common feature (54.0\%). Peritoneal recurrence was more common than hematogenous recurrence $(66.7 \%$ vs. $33.3 \%$ ) among patients with recurrence, and lymphatic recurrence was not observed in our data. Upon comparing characteristics between patients with and without recurrence, age, grade, mitotic count (10 HPF), and type of adjuvant therapy after primary surgery showed significant differences between the 2 groups (Table 1).

Univariate and multivariate analyses were performed to identify prognostic factors for RFS, OS, and survival after recurrence. In analysis for RFS (Table 2), age, stage, tumor suspected as leiomyoma before surgery, residual status after primary surgery, tumor size, grade, nuclear atypia, mitotic count, and history of adjuvant chemotherapy/radiotherapy were significant variables in univariate analysis. Among these factors, age (hazard ratio [HR], 1.091; 95\% Cl, 1.045-1.140; $P<0.001)$, residual disease $(\mathrm{HR}, 5.066 ; 95 \% \mathrm{Cl}, 1.880-$ 13.651; $P<0.001)$, mitotic count ( $>10 / 10 \mathrm{HPF}, \mathrm{HR}, 3.976$; $95 \% \mathrm{Cl}, 1.420-11.131 ; P=0.009)$, and history of radiotherapy $(\mathrm{HR}, 0.209 ; 95 \% \mathrm{Cl}, 0.076-0.578 ; P=0.003)$ were significant variables in multivariate analysis. Fig. $1 A$ and $B$ show Kaplan-Meier curves for the time influenced by significant prognostic factors, residual disease status, and mitotic count. Significant prognostic factors for OS in univariate analysis were stage, residual disease after primary surgery, tumor size, grade, nuclear atypia, mitotic count, and adjuvant chemotherapy (Table 3). In multivariate analysis, the presence of residual disease after primary surgery $(H R, 3.740 ; 95 \%$ $\mathrm{Cl}, 1.170-11.956 ; P=0.026)$ and severe nuclear atypia (HR, $6.041 ; 95 \% \mathrm{Cl}, 1.977-18.465 ; P=0.002)$ were significant prognostic factors associated with OS. Fig. $1 C$ and D show survival curves influenced by residual disease status and nuclear atypia.

For analysis of survival after recurrence, only patients with recurrence were assessed ( $n=30)$. In univariate analysis, nuclear atypia was a significant factor (Table 4). In multivariate analysis, the presence of residual disease status (HR, 11.304; $95 \% \mathrm{Cl}, 2.009-65.598 ; P=0.006)$, severe nuclear atypia (HR, 17.237; 95\% Cl, 2.902-102.384; $P=0.002)$, and hematogenous recurrence $(\mathrm{HR}, 4.189 ; 95 \% \mathrm{Cl}, 1.032-17.000$; $P=0.045$ ) were significantly associated with worse prognosis in survival after recurrence. The survival curve for survival after recurrence is shown in Fig. $1 \mathrm{E}$ and $\mathrm{F}$.

\section{Discussion}

In our study, retrospective analysis of 50 patients with uterine LMS demonstrated possible prognostic factors for RFS, OS, and survival after recurrence. Age, residual disease status, mitotic count, and history of adjuvant radiotherapy were significant factors for RFS. Residual disease status and nuclear atypia were significant factors for OS, while residual disease status, nuclear atypia, and recurrence pattern were significant factors for survival after recurrence. Notably, complete resection of tumor at primary surgery was significantly associated with RFS, OS, and survival after recurrence.

A number of studies on the prognostic factors for uterine LMS have previously been published. The most important prognostic factor for survival in uterine LMS remains tumor stage at diagnosis. In 2009, FIGO presented a new classification for uterine LMS that includes tumor size, extrauterine involvement, and invasion to abdominal tissue [6]. In a previous study of Surveillance, Epidemiology, and End Results data of patients with uterine LMS from 2000 to 2012, almost half of patients were stage I, $14 \%$ were stage II and III, and $31 \%$ were stage IV [13]. Survival outcomes of uterine LMS are poor regardless of stage with 5-year disease-free survival of $65.7 \%$ in total the cohort [14]. In addition to FIGO stage, other reported prognostic factors include age, tumor size, 


\section{Obstetrics \& Gynecology Science}

Vol. 62, No. 2, 2019

Table 1. Characteristics of study cohorts $(n=50)$

\begin{tabular}{|c|c|c|c|c|}
\hline Characteristics & Without recurrence $(n=20)$ & With recurrence $(n=30)$ & $P$-value & All patients $(n=50)$ \\
\hline Age (yr) & $45.5(31-72)$ & $50.5(34-72)$ & 0.041 & $48(31-72)$ \\
\hline Stage & & & 0.149 & \\
\hline । & $15(75.0)$ & $15(50.0)$ & & $30(60.0)$ \\
\hline$\|$ & $1(5.0)$ & $2(6.7)$ & & $3(6.0)$ \\
\hline III & $2(10.0)$ & $2(6.7)$ & & $4(8.0)$ \\
\hline IV & $2(10.0)$ & $11(36.7)$ & & $13(26.0)$ \\
\hline Tumor size $(\mathrm{cm})$ & $7.5(3.0-29.0)$ & $10.0(7.0-22.5)$ & 0.069 & $10.0(3.0-29.0)$ \\
\hline Grade & & & 0.033 & \\
\hline 1 & $5(25.0)$ & $1(3.3)$ & & $6(12.0)$ \\
\hline 2 & $2(10.0)$ & $5(16.7)$ & & $7(14.0)$ \\
\hline 3 & $4(20.0)$ & $15(50.0)$ & & $19(38.0)$ \\
\hline Unknown & $9(45.0)$ & $9(18.0)$ & & $18(36.0)$ \\
\hline Nuclear atypia & & & 0.151 & \\
\hline Mild & $3(15.0)$ & 0 & & $3(6.0)$ \\
\hline Moderate & $11(55.0)$ & $16(53.3)$ & & $27(54.0)$ \\
\hline Severe & $5(25.0)$ & $12(40.0)$ & & $17(34.0)$ \\
\hline Unknown & $1(5.0)$ & $2(6.7)$ & & $3(6.0)$ \\
\hline Mitotic count (/10 HPF) & $10(3-93)$ & $23(3-107)$ & 0.001 & $15(3-107)$ \\
\hline Tumor cell necrosis & $14(70.0)$ & $26(86.7)$ & 0.171 & $40(80.0)$ \\
\hline Residual disease after primary surgery & & & 0.285 & \\
\hline No residual & $18(90.0)$ & $23(76.7)$ & & $41(82.0)$ \\
\hline Residual disease present & $2(10.0)$ & 7 (23.3.) & & $9(18.0)$ \\
\hline $\begin{array}{l}\text { Adjuvant treatment after primary } \\
\text { surgery }\end{array}$ & & & 0.017 & \\
\hline Chemotherapy & $4(20.0)$ & $18(60.0)$ & & $22(44.0)$ \\
\hline Radiotherapy & $6(30.0)$ & $5(16.7)$ & & $11(22.0)$ \\
\hline CCRT & $2(10.0)$ & 0 & & $2(4.0)$ \\
\hline No & $8(40.0)$ & $7(23.3)$ & & $15(30.0)$ \\
\hline Type of adjuvant chemotherapy & & & 0.436 & \\
\hline Doxorubicin-based & $1(25.0)$ & $10(55.6)$ & & $11(50.0)$ \\
\hline Ifosfamide-based & $3(75.0)$ & $7(38.9)$ & & $10(45.5)$ \\
\hline Others & 0 & $1(5.6)$ & & $1(4.5)$ \\
\hline Symptoms & & & 0.276 & \\
\hline No & $6(30.0)$ & $4(13.3)$ & & $10(20.0)$ \\
\hline Vaginal bleeding & $5(25.0)$ & $11(36.7)$ & & $16(32.0)$ \\
\hline Palpable mass & $5(25.0)$ & $12(40.0)$ & & $17(34.0)$ \\
\hline Pelvic pain & $4(20.0)$ & $3(10.0)$ & & $7(14.0)$ \\
\hline $\begin{array}{l}\text { Suspected as leiomyoma before } \\
\text { surgery }\end{array}$ & $8(40.0)$ & $7(23.3)$ & 0.228 & $15(30.0)$ \\
\hline Preoperative serum CA-125 (U/mL) & $12.4(2.65-54.20)$ & $24.8(4.2-294.6)$ & 0.113 & $13.2(2.65-294.6)$ \\
\hline Serum CA-125 at recurrence $(\mathrm{U} / \mathrm{mL})$ & NA & $8.7(2.2-361.1)$ & NA & NA \\
\hline Recurrence pattern & & & $<0.001$ & \\
\hline
\end{tabular}




\section{Obstetrics \& Gynecology Science}

E Sun Paik, et al. Prognosis of uterine leiomyosarcoma

Table 1. Continued

\begin{tabular}{|c|c|c|c|c|}
\hline Characteristics & Without recurrence $(n=20)$ & With recurrence $(n=30)$ & $P$-value & All patients $(n=50)$ \\
\hline No & $20(100.0)$ & & & $20(40.0)$ \\
\hline Peritoneal & & $20(66.7)$ & & $20(40.0)$ \\
\hline Hematogenous & & $10(33.3)$ & & $10(20.0)$ \\
\hline Treatment type at recurrence & & & $<0.001$ & \\
\hline No & $20(100.0)$ & & & $20(40.0)$ \\
\hline Chemotherapy & & $9(30.0)$ & & $9(18.0)$ \\
\hline Radiotherapy & & $15(50.0)$ & & $1(2.0)$ \\
\hline CCRT & & $1(3.3)$ & & $1(2.0)$ \\
\hline Target therapy & & $2(6.7)$ & & $2(4.0)$ \\
\hline Surgical treatment & & $15(50.0)$ & & $15(30.0)$ \\
\hline Conservative & & $2(6.7)$ & & $2(4.0)$ \\
\hline
\end{tabular}

Values are presented as median (interquartile range) or number (\%).

HPF, high-power field; CCRT, concurrent chemo-radiation therapy; CA-125, cancer-antigen 125; NA, not available.

Table 2. Univariate and multivariate Cox proportional hazards analysis for recurrence-free survival to adjust risk associated with prognostic clinical features $(n=50)$

\begin{tabular}{|c|c|c|c|c|}
\hline \multirow{2}{*}{ Variables } & \multicolumn{4}{|c|}{ RFS } \\
\hline & Univariate HR (95\% Cl) & $P$-value & Multivariate HR $(95 \% \mathrm{Cl})$ & $P$-value \\
\hline Age (yr) & $1.05(1.02-1.09)$ & 0.004 & $1.09(1.05-1.14)$ & $<0.001$ \\
\hline Stage (I, II vs. III, IV) & $3.15(1.49-6.68)$ & 0.003 & $0.75(0.19-2.92)$ & 0.682 \\
\hline $\begin{array}{l}\text { Tumor suspected as leiomyoma before } \\
\text { surgery (no vs. yes) }\end{array}$ & $0.39(0.16-0.96)$ & 0.041 & $0.83(0.28-2.46)$ & 0.733 \\
\hline Residual disease (no vs. yes) & $5.06(2.09-12.26)$ & $<0.001$ & $5.07(1.88-13.65)$ & $<0.001$ \\
\hline Size $(\leq 10 \mathrm{~cm}$ vs. $>10 \mathrm{~cm})$ & $2.90(1.33-6.29)$ & 0.007 & $1.25(0.48-3.23)$ & 0.652 \\
\hline Symptoms (no vs. yes) & $2.36(0.82-6.82)$ & 0.112 & & \\
\hline Grade (1, 2 vs. 3) & $2.97(1.36-6.48)$ & 0.006 & $0.49(0.13-1.89)$ & 0.298 \\
\hline Nuclear atypia (mild, moderate vs. severe) & $2.32(1.09-4.95)$ & 0.029 & $2.09(0.77-5.70)$ & 0.150 \\
\hline Mitotic count ( $\leq 10$ vs. $>10 / 10 \mathrm{HPF}$ ) & $3.19(1.28-7.92)$ & 0.013 & $3.98(1.42-11.13)$ & 0.009 \\
\hline Adjuvant chemotherapy (no vs. yes) & $3.72(1.69-8.20)$ & 0.001 & $0.74(0.17-3.20)$ & 0.684 \\
\hline Adjuvant radiotherapy (no vs. yes) & $0.36(0.15-0.89)$ & 0.028 & $0.21(0.08-0.58)$ & 0.003 \\
\hline
\end{tabular}

RFS, recurrence-free survival; $\mathrm{HR}$, hazard ratio; $\mathrm{Cl}$, confidential interval; $\mathrm{HPF}$, high-power field.

mitotic index, and lymphovascular invasion [10,15-18]. Not all of these factors are included in current staging, yet they are related to prognosis.

In a study analyzing prognostic factors using the National Cancer Database [19], surgical resection remained the best effective management for uterine LMS. In the current study, stage-defining variables and other factors were used to analyze prognostic factors, and residual tumor status at primary surgery was also associated with RFS, OS, and survival after recurrence. With regard to surgical resection of uterine
LMS, total hysterectomy with/without bilateral salpingooophorectomy is the standard treatment for patients with uterine-confined disease. Tumor removal should be en bloc, with an effort to avoid intraoperative rupture, morcellation, or spillage in the peritoneal cavity. Despite having metastatic disease, patients with both intraperitoneal and extraperitoneal disease are considered to be appropriate candidates for surgical resection due to limited systemic treatment options. In a previous retrospective study performed at the Memorial Sloan Kettering Cancer Center showed that optimal surgical 


\section{Obstetrics \& Gynecology Science}

Vol. 62, No. 2, 2019

A

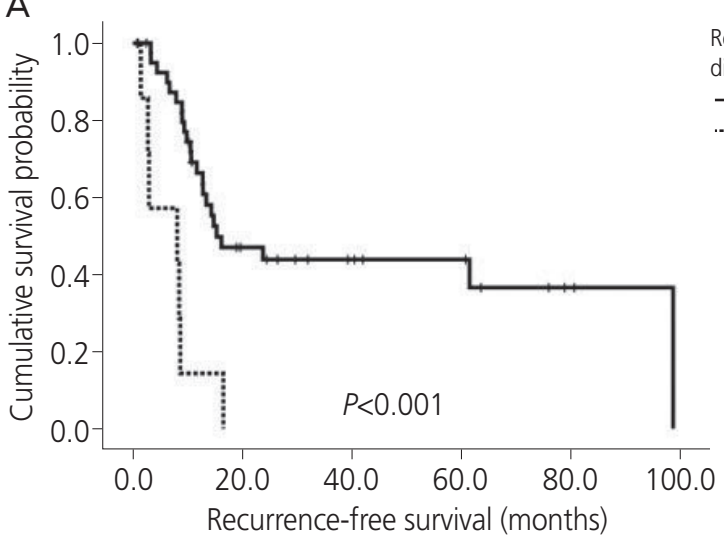

C

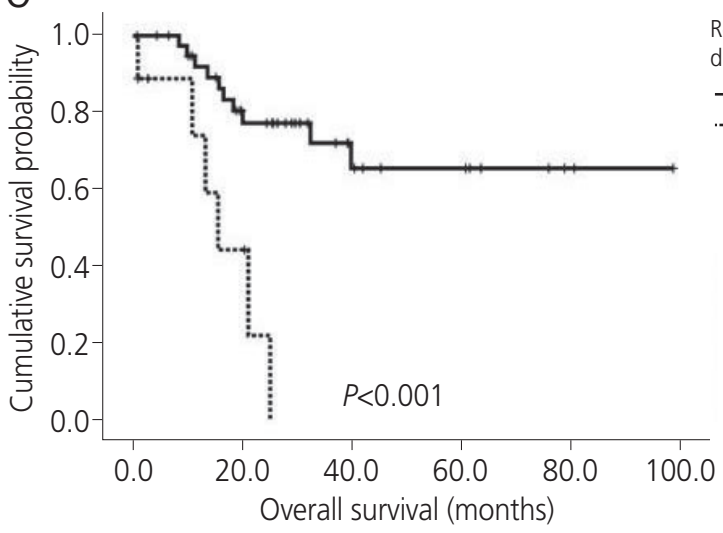

$\mathrm{E}$

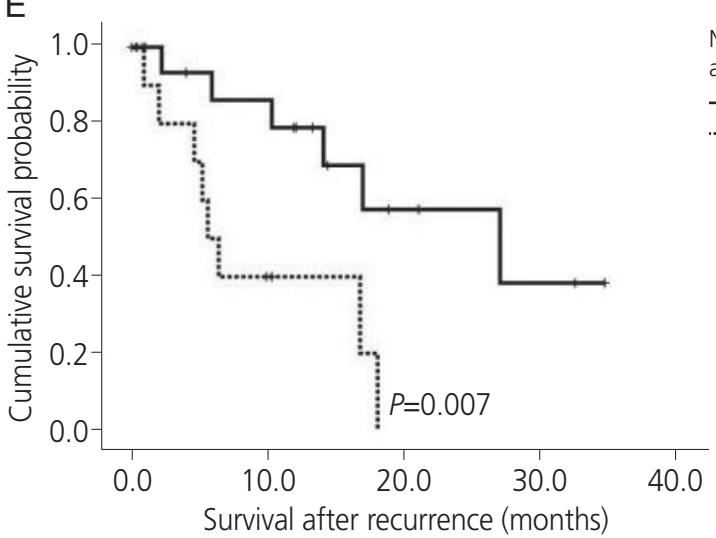

B
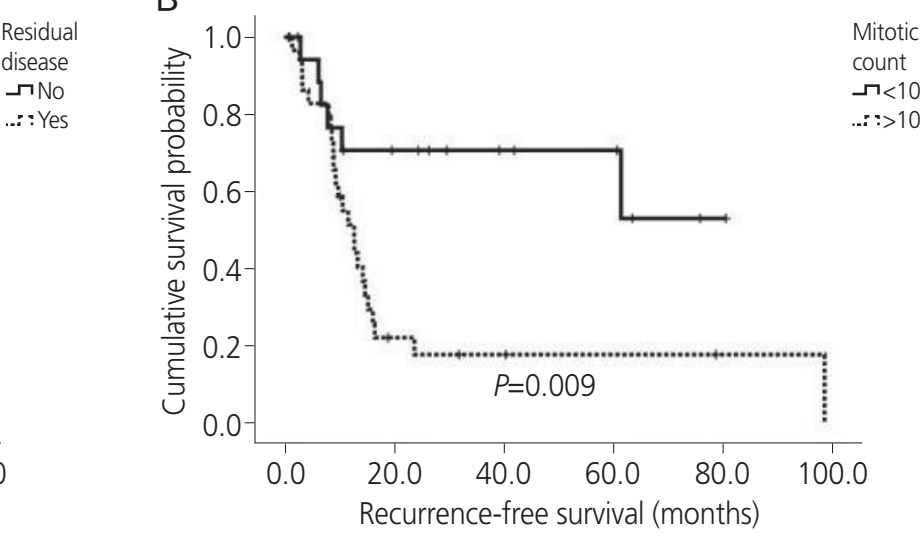

D

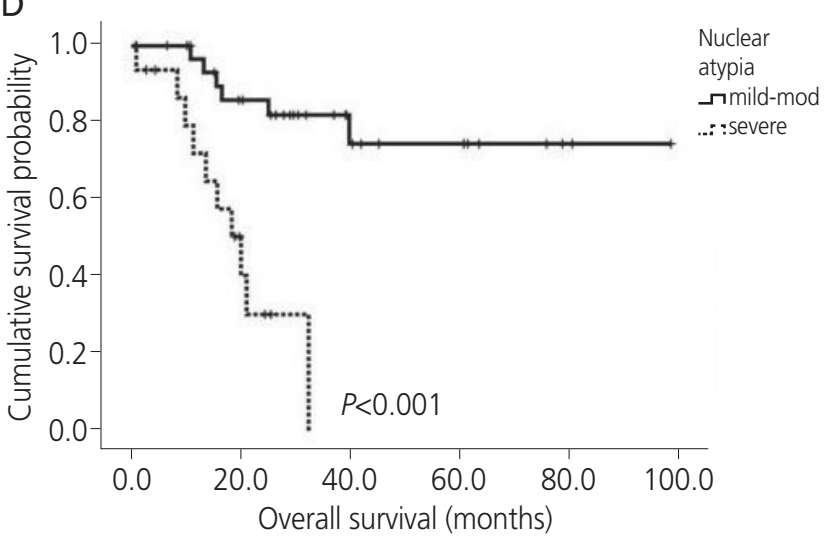

$\mathrm{F}$

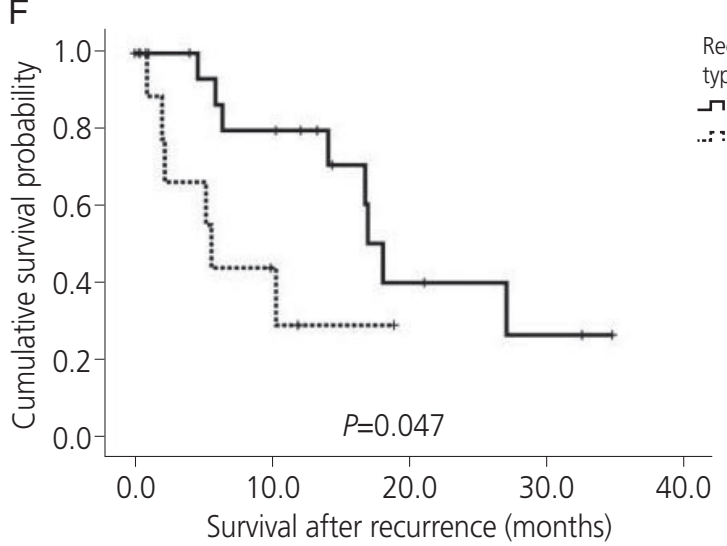

Fig. 1. Kaplan-Meier curve considering the influence of significant prognostic factors for recurrence-free survival $(A, B)$, overall survival (C, $D)$, and survival after recurrence $(E, F)$.

resection was associated with improved progression-free survival [20]. Patients with hematogenous metastasis can also be considered as candidates for surgical resection. Several studies showed improved survival outcomes after pulmonary metastasectomy, and pulmonary metastasectomy is an effective option for selected patients with metastatic uterine LMS [21-24]. According to previous studies, surgical resection for uterine LMS should be performed for better survival outcomes in the appropriate candidates.

For uterine LMS, the effect of adjuvant therapy is still unclear due to its poor efficacy [25]. In our results, administration of adjuvant radiotherapy was associated with improved RFS. However, this was not significant in long-term survival, and survival advantage may not persist. Patients who un- 


\section{Obstetrics \& Gynecology Science}

E Sun Paik, et al. Prognosis of uterine leiomyosarcoma

Table 3. Univariate and multivariate Cox proportional hazards analysis for overall survival to adjust risk associated with prognostic clinical features $(n=50)$

\begin{tabular}{|c|c|c|c|c|}
\hline \multirow{2}{*}{ Variables } & \multicolumn{4}{|c|}{ OS } \\
\hline & Univariate HR (95\% Cl) & $P$-value & Multivariate HR (95\% Cl) & $P$-value \\
\hline Age (yr) & $1.07(0.99-1.08)$ & 0.108 & NA & NA \\
\hline Stage (I, II vs. III, IV) & $3.69(1.35-10.10)$ & 0.011 & $0.64(0.11-3.74)$ & 0.618 \\
\hline $\begin{array}{l}\text { Tumor suspected as leiomyoma before } \\
\text { surgery (no vs. yes) }\end{array}$ & $0.27(0.06-1.19)$ & 0.083 & $0.45(0.08-2.53)$ & 0.454 \\
\hline Residual disease (no vs. yes) & $6.08(2.08-17.83)$ & 0.001 & $3.74(1.17-11.96)$ & 0.026 \\
\hline Size $(\leq 10 \mathrm{~cm}$ vs. $>10 \mathrm{~cm})$ & $3.73(1.37-10.19)$ & 0.010 & $0.90(0.23-3.60)$ & 0.881 \\
\hline Symptoms (no vs. yes) & $0.57(0.27-1.20)$ & 0.137 & NA & NA \\
\hline Grade (1, 2 vs. 3$)$ & $4.39(1.55-12.44)$ & 0.005 & $0.76(0.17-3.39)$ & 0.720 \\
\hline Nuclear atypia (mild, moderate vs. severe) & $7.36(2.41-22.42)$ & $<0.001$ & $6.04(1.98-18.47)$ & 0.002 \\
\hline Mitotic count ( $\leq 10$ vs. $>10 / 10$ HPF) & $3.57(1.01-12.70)$ & 0.049 & $1.55(0.32-7.59)$ & 0.586 \\
\hline Adjuvant chemotherapy (no vs. yes) & $5.53(1.77-17.24)$ & 0.003 & $2.86(0.82-10.05)$ & 0.132 \\
\hline Adjuvant radiotherapy (no vs. yes) & $0.44(0.13-1.54)$ & 0.198 & NA & NA \\
\hline
\end{tabular}

OS, overall survival; HR, hazard ratio; Cl, confidential interval; HPF, high-power field; NA, not available.

Table 4. Univariate and multivariate Cox proportional hazards analysis for survival after recurrence to adjust risk associated with prognostic clinical features $(n=30)$

\begin{tabular}{|c|c|c|c|c|}
\hline \multirow{2}{*}{ Variables } & \multicolumn{4}{|c|}{ Survival after recurrence } \\
\hline & Univariate HR (95\% Cl) & $P$-value & Multivariate HR $(95 \% \mathrm{Cl})$ & $P$-value \\
\hline Age (yr) & $1.00(0.96-1.06)$ & 0.868 & NA & NA \\
\hline Stage (I, II vs. III, IV) & $1.99(0.64-6.12)$ & 0.232 & NA & NA \\
\hline $\begin{array}{l}\text { Tumor suspected as leiomyoma before } \\
\text { surgery (no vs. yes) }\end{array}$ & $0.92(0.20-4.18)$ & 0.915 & NA & NA \\
\hline Residual disease (no vs. yes) & $2.64(0.83-8.40)$ & 0.099 & $11.30(2.01-63.60)$ & 0.006 \\
\hline Size $(\leq 10 \mathrm{~cm}$ vs. $>10 \mathrm{~cm})$ & $1.25(0.43-3.59)$ & 0.684 & NA & NA \\
\hline Symptoms (no vs. yes) & $2.26(0.48-10.71)$ & 0.304 & NA & NA \\
\hline Grade (1, 2 vs. 3$)$ & $1.96(0.65-5.88)$ & 0.232 & NA & NA \\
\hline Nuclear atypia (mild, moderate vs. severe) & $4.29(1.37-13.46)$ & 0.012 & $17.24(2.90-102.38)$ & 0.002 \\
\hline Mitotic count ( $\leq 10$ vs. $>10 / 10$ HPF) & $6.26(0.78-50.30)$ & 0.085 & $4.12(0.39-43.88)$ & 0.240 \\
\hline $\begin{array}{l}\text { Recurrence type (peritoneal vs. } \\
\text { hematogenous) }\end{array}$ & $2.97(0.97-9.09)$ & 0.057 & $4.12(1.03-17.00)$ & 0.045 \\
\hline $\begin{array}{l}\text { Treatment type at recurrence (non-surgical } \\
\text { treatment vs. surgical treatment) }\end{array}$ & $0.35(0.12-1.07)$ & 0.065 & $0.38(0.09-1.67)$ & 0.200 \\
\hline
\end{tabular}

HR, hazard ratio; $\mathrm{Cl}$, confidential interval; HPF, high-power field; NA, not available.

derwent adjuvant chemotherapy showed poor survival in univariate analysis for RFS and OS, but not in multivariate analysis, probably because chemotherapy is for patients with advanced stage or distant metastasis. We cannot further support results regarding adjuvant therapy because the results are based on a small number of patients.

Diagnostic evaluation of uterine LMS and distinguishing between benign leiomyoma and LMS is difficult because they often have similar symptoms. Abnormal uterine bleeding, pelvic pain or palpation of abdominal mass may present in both diseases. Also, there are currently no validated clinical or radiologic criteria to differentiate between leiomyoma and LMS, as the final diagnosis is made histopathologically after surgery. In our analysis, we determined whether a tumor sus- 


\section{Obstetrics \& Gynecology Science}

Vol. 62, No. 2, 2019

pected as LMS before surgery with clinical characteristics can be a useful prognostic factor in LMS. It was only significant in univariate analysis for RFS, but not in multivariate analysis. It was not significantly related to survival in our results. With respect to tumor marker for LMS, serum lactate dehydrogenase level is known to be elevated in some patients [26]. In our study, we were able to analyze only serum cancer antigen (CA)-125 levels due to limited data and the retrospective nature of this study. There was a median initial serum CA125 level difference between the recurrent and non-recurrent group, but it was not statistically significant. Serum CA125 elevation was not observed at relapse. We did not find a relationship between CA-125 level and the prognosis of LMS patients from our data, which is similar to a previous study that showed that CA-125 was not immunohistochemically expressed in LMS tumors [27].

This study has some limitations. The analysis was performed on the basis of retrospective data, with the lack of detailed medical histories, short-term clinical outcomes, and detailed data on treatments after recurrence that could be related to prognosis. Because uterine LMS is a rare disease and analysis was performed with data from a single institution, the number of patients is smaller in this study than in other studies. A small number of cases may have caused an overfitting problem in multivariate analysis and may have reduced the accuracy of analysis. Race is known to be associated with differences in survival [14], but our study included patients who were almost all Asians. Despite the drawbacks of confounders and biases, we have drawn some valid conclusions from this limited data. This study suggests possible prognostic factors for not only RFS and OS but also survival after recurrence.

Further research should focus on the evaluation of patient counseling alternatives respect to these results. This information may be useful for counseling patients with uterine LMS. Also, research on developing improved technology to differentiate between benign and malignant uterine diseases should be performed to enable quicker surgical intervention for better prognosis. Additionally, the development of novel therapeutics for uterine LMS with poor prognosis is needed.

In conclusion, we demonstrated the possible prognostic factors for recurrence, OS, and survival after recurrence based on data from LMS patients treated at a single institution. Despite some limitations, these results provide useful information for patients with LMS.

\section{Acknowledgements}

This research was supported by the Sungkyunkwan Research Fund, Sungkyunkwan University, 2017.

\section{Conflict of Interest}

No potential conflict of interest relevant to this article was reported.

\section{Ethical approval}

The study was approved by the Institutional Review Board of Samsung Medical Center (IRB No. 2018-03-103-001) and performed in accordance with the principles of the Declaration of Helsinki. Written informed consents were obtained.

\section{Patient consent}

Informed consent was waived because of the retrospective study design.

\section{References}

1. Toro JR, Travis LB, Wu HJ, Zhu K, Fletcher CD, Devesa SS. Incidence patterns of soft tissue sarcomas, regardless of primary site, in the surveillance, epidemiology and end results program, 1978-2001: an analysis of 26,758 cases. Int J Cancer 2006;119:2922-30.

2. Felix AS, Cook LS, Gaudet MM, Rohan TE, Schouten LJ, Setiawan VW, et al. The etiology of uterine sarcomas: a pooled an]alysis of the epidemiology of endometrial cancer consortium. Br J Cancer 2013;108:727-34.

3. Foley OW, Rauh-Hain JA, Clemmer J, Clark RM, Hall T, Diver EJ, et al. Trends in the treatment of uterine leiomyosarcoma in the Medicare population. Int J Gynecol Cancer 2015;25:453-8.

4. D'Angelo E, Prat J. Uterine sarcomas: a review. Gynecol Oncol 2010;116:131-9.

5. Kim WY, Chang SJ, Chang KH, Yoon JH, Kim JH, Kim $\mathrm{BG}$, et al. Uterine leiomyosarcoma: 14-year two-center 


\section{Obstetrics \& Gynecology Science}

E Sun Paik, et al. Prognosis of uterine leiomyosarcoma

experience of 31 cases. Cancer Res Treat 2009;41:24-8.

6 . Prat J. FIGO staging for uterine sarcomas. Int J Gynaecol Obstet 2009;104:177-8.

7. Leibsohn S, d'Ablaing G, Mishell DR Jr, Schlaerth JB. Leiomyosarcoma in a series of hysterectomies performed for presumed uterine leiomyomas. Am J Obstet Gynecol 1990;162:968-74.

8. Leitao MM, Sonoda Y, Brennan MF, Barakat RR, Chi DS. Incidence of lymph node and ovarian metastases in leiomyosarcoma of the uterus. Gynecol Oncol 2003;91:209-12.

9. Schwartz Z, Dgani R, Lancet M, Kessler I. Uterine sarcoma in Israel: a study of 104 cases. Gynecol Oncol 1985;20:354-63.

10. Zivanovic O, Jacks LM, lasonos A, Leitao MM Jr, Soslow RA, Veras $E$, et al. A nomogram to predict postresection 5-year overall survival for patients with uterine leiomyosarcoma. Cancer 2012;118:660-9.

11. Kempson RL, Bari W. Uterine sarcomas. Classification, diagnosis, and prognosis. Hum Pathol 1970;1:331-49.

12. Hosmer DW Jr, Lemeshow S, Sturdivant RX. Applied logistic regression. 3rd ed. Hoboken (NJ): John Wiley \& Sons; 2013.

13. Hosh M, Antar S, Nazzal A, Warda M, Gibreel A, Refky B. Uterine sarcoma: analysis of 13,089 cases based on Surveillance, Epidemiology, and End Results Database. Int J Gynecol Cancer 2016;26:1098-104.

14. Kapp DS, Shin JY, Chan JK. Prognostic factors and survival in 1396 patients with uterine leiomyosarcomas: emphasis on impact of lymphadenectomy and oophorectomy. Cancer 2008;112:820-30.

15. Pelmus M, Penault-Llorca F, Guillou L, Collin F, Bertrand $G$, Trassard $M$, et al. Prognostic factors in early-stage leiomyosarcoma of the uterus. Int J Gynecol Cancer 2009;19:385-90.

16. Abeler VM, Røyne O, Thoresen S, Danielsen HE, Nesland $J M$, Kristensen GB. Uterine sarcomas in Norway. A histopathological and prognostic survey of a total population from 1970 to 2000 including 419 patients. Histopathology 2009;54:355-64.

17. Dinh TA, Oliva EA, Fuller AF Jr, Lee H, Goodman A. The treatment of uterine leiomyosarcoma. Results from a 10-year experience (1990-1999) at the Massachusetts
General Hospital. Gynecol Oncol 2004;92:648-52.

18. Mayerhofer K, Obermair A, Windbichler G, Petru E, Kai$\operatorname{der} A$, Hefler $L$, et al. Leiomyosarcoma of the uterus: a clinicopathologic multicenter study of 71 cases. Gynecol Oncol 1999;74:196-201.

19. Seagle BL, Sobecki-Rausch J, Strohl AE, Shilpi A, Grace A, Shahabi S. Prognosis and treatment of uterine leiomyosarcoma: a National Cancer Database study. Gynecol Oncol 2017;145:61-70.

20. Leitao MM Jr, Zivanovic O, Chi DS, Hensley ML, O'Cearbhaill R, Soslow RA, et al. Surgical cytoreduction in patients with metastatic uterine leiomyosarcoma at the time of initial diagnosis. Gynecol Oncol 2012;125:409-13.

21. Anderson TM, McMahon JJ, Nwogu CE, Pombo MW, Urschel JD, Driscoll DL, et al. Pulmonary resection in metastatic uterine and cervical malignancies. Gynecol Oncol 2001;83:472-6.

22. Clavero JM, Deschamps C, Cassivi SD, Allen MS, Nichols FC 3rd, Barrette BA, et al. Gynecologic cancers: factors affecting survival after pulmonary metastasectomy. Ann Thorac Surg 2006;81:2004-7.

23. Leitao MM, Brennan MF, Hensley M, Sonoda $Y$, Hummer A, Bhaskaran D, et al. Surgical resection of pulmonary and extrapulmonary recurrences of uterine leiomyosarcoma. Gynecol Oncol 2002;87:287-94.

24. Paik ES, Yoon A, Lee YY, Kim TJ, Lee JW, Bae DS, et al. Pulmonary metastasectomy in uterine malignancy: outcomes and prognostic factors. J Gynecol Oncol 2015;26:270-6.

25. Greer BE, Koh WJ, Abu-Rustum N, Bookman MA, Bristow RE, Campos SM, et al. Uterine neoplasms. Clinical practice guidelines in oncology. J Natl Compr Canc Netw 2009;7:498-531.

26. Nishigaya Y, Kobayashi Y, Matsuzawa Y, Hasegawa K, Fukasawa I, Watanabe $Y$, et al. Diagnostic value of combination serum assay of lactate dehydrogenase, D-dimer, and C-reactive protein for uterine leiomyosarcoma. J Obstet Gynaecol Res 2019;45:189-94.

27. Menczer J, Schreiber L, Berger E, Ben-Shem E, Golan A, Levy T. CA125 expression in the tissue of uterine leiomyosarcoma. Isr Med Assoc J 2014;16:697-9. 